\title{
TITLE:
}

\section{Creep behavior of bamboo under various desorption conditions}

\section{$\operatorname{AUTHOR(S):~}$}

Tsubaki, Takashi; Nakano, Takato

CITATION:

Tsubaki, Takashi ... [et al]. Creep behavior of bamboo under various desorption conditions. Holzforschung 2010, 64(4): 489-493

ISSUE DATE:

2010-06

URL:

http://hdl.handle.net/2433/141946

RIGHT:

(C) 2007-2011. Walter de Gruyter GmbH \& Co. KG. 


\section{Creep behavior of bamboo under various desorption conditions}

\author{
Takashi Tsubaki and Takato Nakano* \\ Laboratory of Biomaterials Design, Division of Forest and \\ Biomaterials Science, Graduate School of Agriculture, \\ Kyoto University, Kyoto, Japan \\ *Corresponding author. \\ Laboratory of Biomaterials Design, Division of Forest and \\ Biomaterials Science, Graduate School of Agriculture, Kyoto \\ University, Kyoto, Japan \\ E-mail: tnakano@kais.kyoto-u.ac.jp
}

\begin{abstract}
Longitudinal bending creep during desorption, which is also referred to as mechano-sorptive creep, was measured under various desorption conditions for moso bamboo (Phyllostachys pubescens) to clarify the effect of the conditions and the specimen part. The results showed that the mechanosorptive effect appeared not only in wood but also in bamboo, and that its effect was reduced by an increase in the volume fraction of the bundle sheath in the cross-sectional area. The results of this experiment suggest that mechanosorptive behavior is related to the amorphous matrix in bamboo components.
\end{abstract}

Keywords: bamboo; creep; mechano-sorptive; vascular bundle; water desorption.

\section{Introduction}

Wood and bamboo are biological materials for which their mechanical properties in general, and their mechanical relaxation attributes in particular, are closely related to moisture content (MC). Mechanical relaxation under variable moisture conditions is the most interesting property of all (mechanosorptive creep). Grossman (1976) studied the phenomenon for wood. There are two typical behaviors in this regard. The first is considerable relaxation compared to that of constant moisture conditions concerning the initial adsorption or desorption processes, and second is its recovery during adsorption following desorption. Many hypotheses have been postulated about its mechanisms (Schniewind 1966; Takemura 1967, 1968; Leicester 1971; Hunt 1984; Mukudai and Yata 1986, 1987; Hunt and Shelton 1987, 1988; van der Put 1989; Nakano 1996; Tokumoto et al. 1998). The underlying mechanisms, however, are not yet completely understood.

The highly ordered structure of bamboo is much simpler than that of wood. Bamboo consists mainly of the vascular bundle and parenchyma parallel to the longitudinal direction. The vascular bundle is sclerenchymatous fiber that makes the bamboo framework rigid, whereas parenchyma is soft cell that holds nutrients. The latter has different mechanical properties from the former. Therefore, the ratio of the vascular bundle to parenchyma is a very important factor for the mechanical properties in the longitudinal direction. The degree of relaxation should be related to the volume fraction of the vascular bundle in bamboo material.

Chuma et al. (1990, 1991) and Ario et al. (2003) reported that the mechanical behavior of bamboo with constant MC could be analyzed based on the law of mixture, whereby bamboo was regarded as a complex of the vascular bundle and parenchyma. Aoyagi and Nakano (2009) examined the creep behavior of bamboo at constant MC and showed that the relationship between logarithmic compliance and logarithmic time was represented by Nutting's equation (Nutting 1921). They also found that the degree of relaxation decreased with an increase in the volume fraction of the vascular bundle, i.e., the creep behavior of bamboo was governed by the ratio of the vascular bundle to parenchyma.

The contribution of the vascular bundle or parenchyma and the relaxation response to changes in MC are important factors in understanding the mechanisms of mechano-sorptive behavior. However, fewer studies have been published on bamboo compared to wood. Inokuchi et al. (2002) examined the relaxation of bamboo during desorption. They found that bamboo shows a remarkable relaxation during desorption, similar to that of wood, and that this relaxation is related to the volume fraction of both the bundle sheath and water extractives. These authors, however, did not discuss the contribution of $\mathrm{MC}$, the effects of desorption conditions, or the volume fraction of the vascular bundle in detail. More data from diverse viewpoints are required to clarify the mechanisms of the mechano-sorptive relaxation process. Bamboo is more suitable as a model for mechano-sorptive behavior than wood because of its simple microstructure.

In this paper, the creep of bamboo was measured during desorption, and the effects of the high-order structure and desorption conditions were examined on creep behavior. The mechanisms of mechano-sorptive behavior are discussed based on these results.

\section{Materials and methods}

Specimens were prepared from 4-year-old moso bamboo (Phyllostachys heterocycla f. pubescens) with 27 internodes. Fifth and sixth internodes counting from the bottom of the column were investi- 
gated. Specimens were taken from the outer and inner side of the center in a cross-section after removing the epidermis and inner bark. The rectangular dimensions of each specimen were 90 $(\mathrm{L}) \times 1.5(\mathrm{R}) \times 8(\mathrm{~T}) \mathrm{mm}$. The specimens were subjected to two boiling treatments for $30 \mathrm{~min}$ under atmospheric pressure after being injected with distilled water under vacuum to release the internal stress. A gradual air-drying followed at room temperature for 1 week with further drying at $60^{\circ} \mathrm{C}$ under vacuum for 1 day. The oven-dry weights and dimensions of the dried specimens were measured. The average density of the oven-dry specimens was $0.44 \mathrm{~g} / \mathrm{cm}^{3}$ for inner side specimens and $0.78 \mathrm{~g} / \mathrm{cm}^{3}$ for outer side specimens. Considering that the report by Chuma et al. (1990) showed that the volume fraction of vascular bundle was proportional to density, we can estimate from the difference of density that the volume fraction of vascular bundle in the outer region is higher than in the inner region (Figure 1).

Specimens were kept in desiccators at $23^{\circ} \mathrm{C}$ for 3 weeks to achieve different levels of relative humidity (RH): $53 \%, 62 \%, 75 \%$, $84 \%$, and $92 \%$. At the end of this process, the MC of the specimens was in the range of $0.05-0.15$. In this study, one specimen was measured under each desorption condition. The repeatability of creep behavior experiments was confirmed for multiple specimens at $92 \% \mathrm{RH}$. Thus, it is clear that the systematic results are valid.

Creep tests were conducted in three-point center concentrated load bending with a 70-mm span. The load was less than one-third of the proportional limit load for the specimen conditioned at $92 \%$ $\mathrm{RH}$, which was applied to the LT plane along the direction from the outer side face to the inner side of the specimen. The load was $600 \mathrm{~g}$ for the outer side specimens and $150 \mathrm{~g}$ for the inner side. The creep during desorption was quantified in a chamber at $23^{\circ} \mathrm{C}$ and $20 \% \mathrm{RH}$. Another specimen with the same initial MC was simultaneously weighed with an electrical balance in the same chamber. The measurement period was approximately $24 \mathrm{~h}$ because specimen MC almost leveled off. The temperature change in the chamber during these tests was less than $\pm 1{ }^{\circ} \mathrm{C}$, and the humidity change was less than $\pm 3 \%$. The creep of a specimen with constant

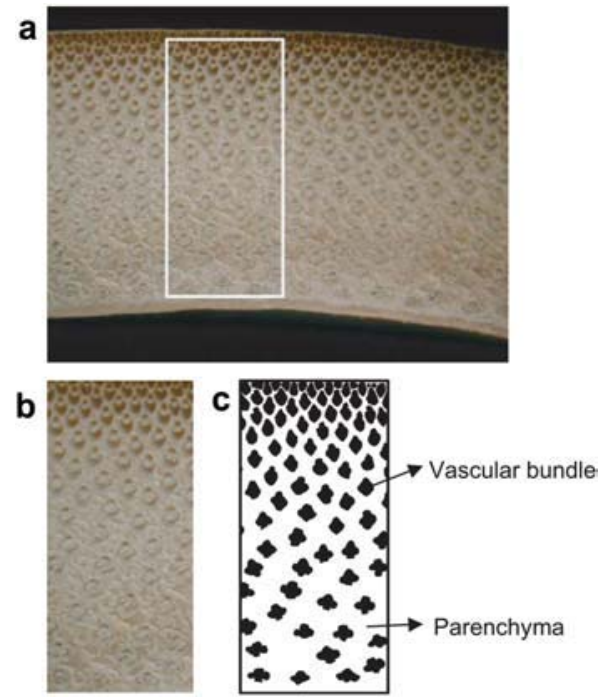

Figure 1 Macroscopic structure of a cross-section of moso bamboo.

(a) General view of a $9(\mathrm{R}) \times 15$ (T) mm section; (b) an enlarged view of the rectangle zone in (a); and (c) a view after binary treatment of (b). The upper part of the image corresponds to the outer side.
MC was also measured in the same chamber; this specimen was wrapped in polyethylene film to keep the change in MC below -0.005 . A linear regression curve was obtained by plotting the dimensions versus $\mathrm{MC}$ in each direction under initial, finished, and oven-dried conditions. Creep compliance during desorption was corrected by means of this regression curve concerning the dimension change of specimens during the test.

\section{Results and discussion}

Figure 2 shows the creep compliance as a function of $\ln (t)$ for the outer and inner side specimens with various constant MCs. The creep compliance increased with the increase in $\mathrm{MC}$ for both specimens, and the degree of relaxation for the inner side was greater than that for the outer side. The relaxation of bamboo is smaller than that of wood. In general, longitudinal creep compliance of moist or wet wood is increased by $100 \%$ during $24 \mathrm{~h}$ (Schniewind 1966), whereas bamboo with MCs investigated in this study shows a 5-20\% increase (Aoyagi and Nakano 2009). Results presented in Figure 2 are in agreement with those of the latter quoted work.

Figure 3a (inner side) and b (outer side) show creep compliance and $\mathrm{MC}$ versus $\ln (\mathrm{t})$ during desorption over the range of various initial MCs. The compliance change of the inner side specimens was greater than that of the outer side spec-


Figure 2 Creep of the outer and inner side specimens with various moisture contents under constant moisture conditions. 

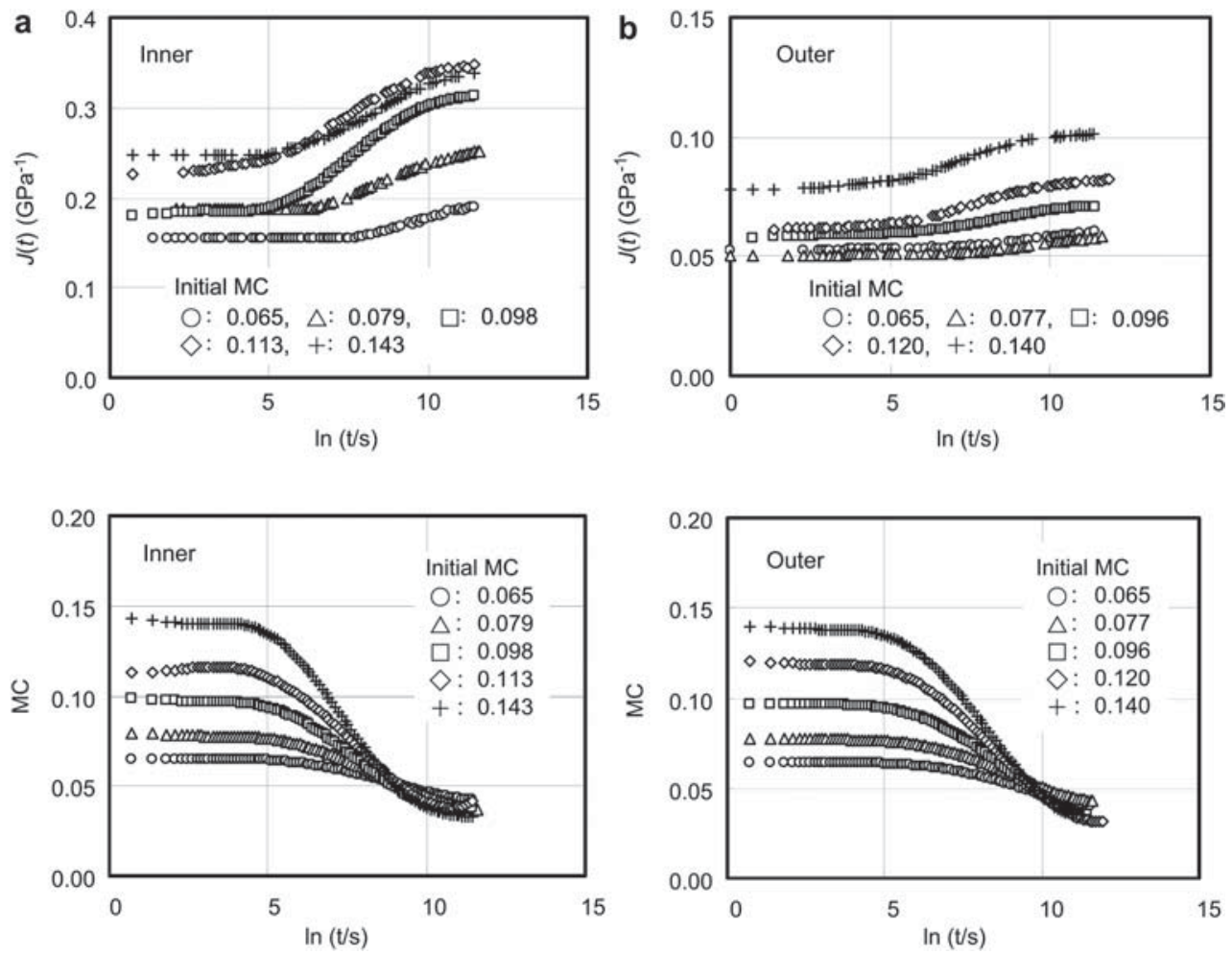

Figure 3 Creep of bamboo specimens with various moisture contents during desorption. (a) Inner part, (b) outer part (see Figure 1).

imens. This implies that a higher volume fraction of vascular bundle results in a lower creep deformation, as previously reported by Inokuchi et al. (2002) and Aoyagi and Nakano (2009). Note that a higher initial MC resulted in a greater compliance change.

Figure 4 shows the MC change in the measurements of Figure 3. The relationship between the maximum desorption rate and the initial MC was linear for both the outer and inner side specimens. The relationship between the time of the maximum rate and the initial $\mathrm{MC}$ was also linear, and it was different for the inner part and the outer part of the specimen with the time of the maximum desorption rate being less for the former than for the latter. This means that the desorption rate of the inner part reached its maximum more quickly.

We compared the creep compliances for the outer and inner side specimens with approximately the same initial MC to analyze the difference in creep behavior between a constant MC process and desorption process. The instantaneous creep compliance values at $\ln (t)=0$, i.e., $t=1 \mathrm{~s}$ were nearly equal for specimens with the same initial MC. Figure 5 shows a comparison of the creep behaviors for two different initial MCs for the outer and inner side specimens. In Figure 5 , the creep compliance during the desorption process was greater than that under the constant MC condition. It is also visible that the difference in creep compliance between the constant MC condition and the desorption process was greater for the inner than that for the outer side specimens. We suggest that the mechano-sorptive effect is as a result of the parenchyma, not the vascular bundle.
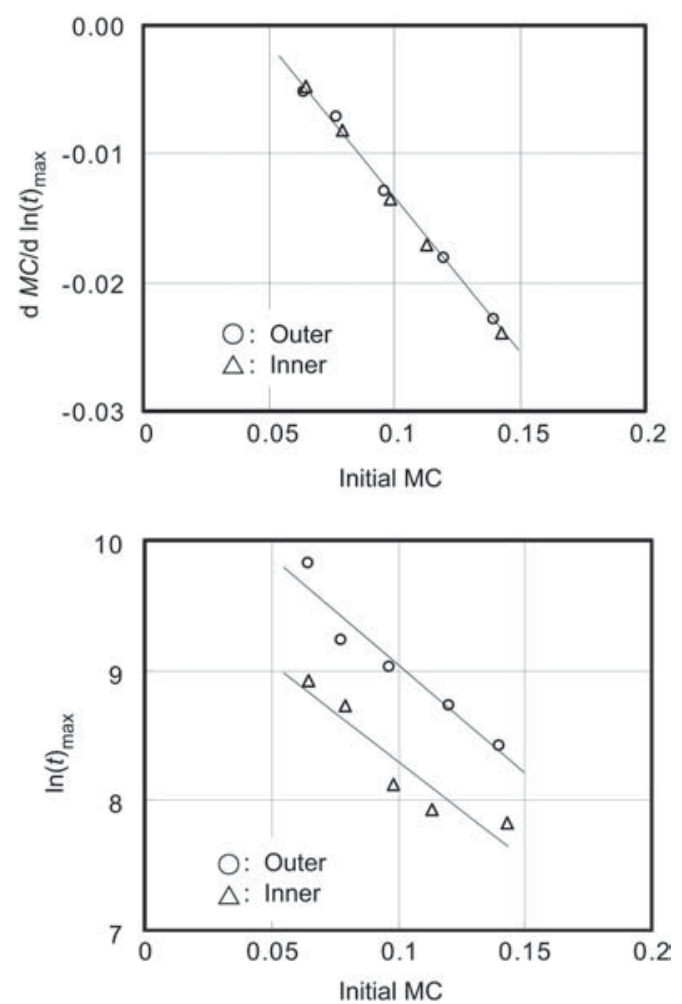

Figure 4 Dependence of $\mathrm{d} M C / \mathrm{d} \ln (t)_{\max }$ and $\ln (t)_{\max }$ on the initial MC.

Note: $\mathrm{d} M C / \mathrm{d} \ln (t)_{\max }$, the maximum desorption rate; $\ln (t)_{\max }$, the logarithm of that time. 



Figure 5 Comparison of creep at a constant moisture content with that during desorption.

The difference in creep behavior between the constant MC condition and the desorption process was compared based on the values of creep compliance at $t=10^{4} \mathrm{~s}$, as presented in Figure 6. The $10^{4} \mathrm{~s}$ is adopted as the time that mechanosorptive effect appeared satisfactory. The plots should be on the line $y=x$ when both creep compliances are equal. For the outer side specimens, the plots were almost on $y=x$, i.e., the mechano-sorptive effect was not effective. In contrast, the plots were far from $y=x$ for the inner side specimens; this was particularly evident for MCs greater than 0.09. Hailwood and Horrobin (1946) reported that this MC level is near the lower limit value for the dissolved water of wood applying the Hailwood and Horrobin theory, which is closely related to swelling.

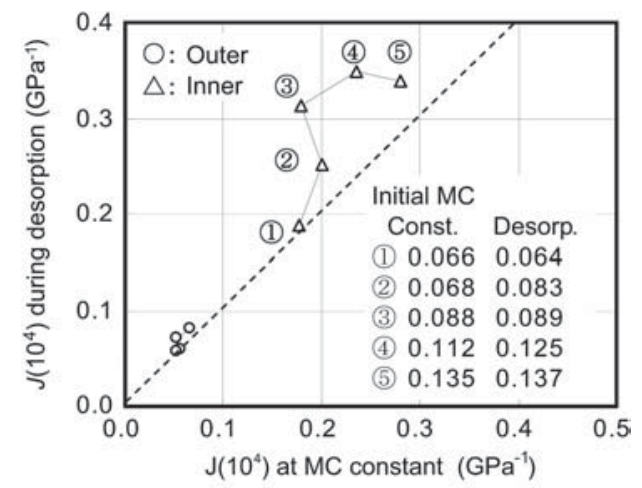

Figure 6 Comparison of creep compliance at $10^{4} \mathrm{~s}$ for a constant moisture content with that during desorption.
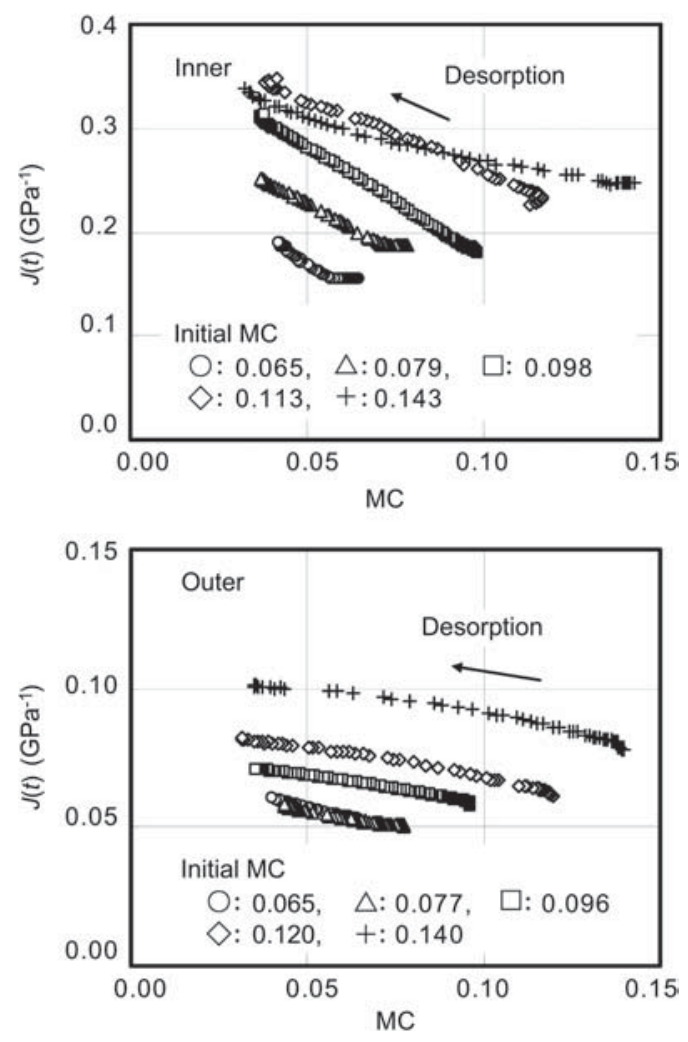

Figure 7 Relationships between creep compliance and moisture content for creep during desorption.

These results show that the mechano-sorptive effect is present not only in wood but also in bamboo and that it is dependent on the fine structure of bamboo and occurs particularly in the parenchyma and not in vascular bundle.

In general, we must consider two factors in the relaxation process under changing moisture conditions: the high-order structure and the MC change of a specimen. In this regard, Hunt (1984) and Hunt and Shelton $(1987,1988)$ pointed out the importance of considering the relationship between creep compliance and $\mathrm{MC}$ in the analysis of mechano-sorptive creep. No descriptions of this relationship for bamboo have been reported, but many studies have shown that it was linear for wood.

Figure 7 shows that the relationship between the creep compliance and the MC in this study appears to be linear like that of wood during desorption. The slope depends on the initial MC, but the dependence is clearly different in Figure 7 for the outer and inner side specimens. Figure 8 shows the relationship between the initial MC and the slope calculated from Figure 7. The absolute values of the slope for the outer side specimens were very low and independent on the initial MC, unlike those for the inner side specimens. More specifically, the absolute values for the inner side specimens decreased as the initial MC increased. Considering the relationship between the initial $\mathrm{MC}$ and the maximum desorption rate shown in Figure 4, this means that larger maximum desorption rates $\mathrm{d} M C / \mathrm{d} \ln (\mathrm{t})_{\max }$ were related to lower creep compliance rates with $\mathrm{MC} \mathrm{d} J(\mathrm{t}) / \mathrm{d} M C$. 


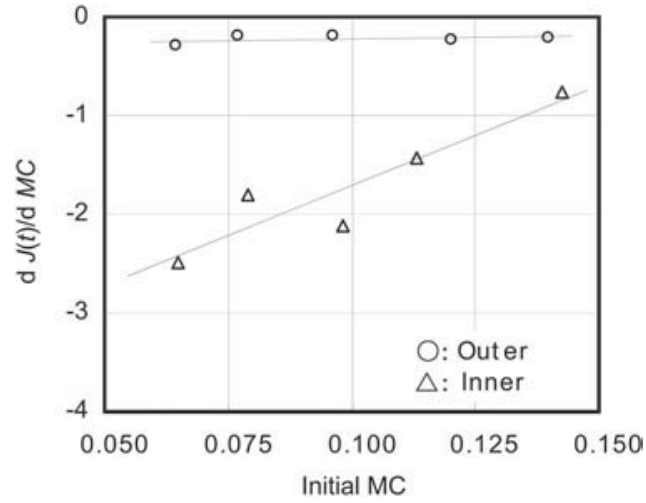

Figure 8 Dependence of $\mathrm{d} J(t) / \mathrm{d} M C$ on the initial MC for the outer and inner side specimens.

Note: $\mathrm{d} J(t) / \mathrm{d} M C$ is the slope in Figure 7.

\section{Conclusions}

The effects of the fine structure and desorption conditions on creep compliance were examined on the basis of creep behavior under various desorption conditions for moso bamboo. A mechano-sorptive behavior was observed. This effect was more pronounced in the inner part of the bamboo, which is rich in parenchyma. The effect decreased as the ratio of the vascular bundle to parenchyma increased. The results demonstrate that the parenchymatic cells are the key components involved in mechano-sorptive behavior. The bundle sheath containing the vascular bundle had little influence on the mechano-sorptive effect. Based on these results one could assume that the mechano-sorptive effect of wood is also closely related to the amorphous matrix in the wood components. Our future research will include the study of bamboo creep during desorption-adsorption cycle testing.

\section{References}

Aoyagi, S., Nakano, T. (2009) Effect of longitudinal and radial position on creep for bamboo. J. Soc. Mat. Sci. Jpn. 58:57-61 (in Japanese).

Ario, I., Morita, C., Suyama, H., Sato, E., Fujii, K. (2003) Mechanical considerations of the laminated composite structure modeled on the anisotropic organization of a bamboo. Trans. Jpn. Soc. Mech. Eng. 69:148-153 (in Japanese).

Chuma, S., Hirohashi, M., Ohgama, T., Kasahara, Y. (1990) Composite structure and tensile properties of mousou bamboo. J. Soc. Mat. Sci. Jpn. 39:847-851 (in Japanese).
Chuma, S., Hirohashi, M., Ohgama, T., Kasahara, Y. (1991) Composite structure and mechanical property of mousou bamboo (Young's modulus in tangential direction of cylindrical wall). J. Soc. Mat. Sci. Jpn. 40:21-26 (in Japanese).

Grossman, P.U.A. (1976) Requirements for a model that exhibits mechano-sorptive behavior. Wood Sci. Technol. 10:163-168.

Hailwood, A.J., Horrobin, S. (1946) Absorption of water by polymers: analysis in terms of a simple model. Trans. Faraday Soc. 42:B084-B092.

Hunt, D.G. (1984) Creep trajectories for beech during moisture changes under load. J. Mater. Sci. 19:1456-1467.

Hunt, D.G., Shelton, C.F. (1987) Stable-state creep limit of softwood. J. Mater. Sci. Lett. 6:353-354.

Hunt, D.G., Shelton, C.F. (1988) Longitudinal moisture-shrinkage coefficients of softwood at the mechano-sorptive creep limit. Wood Sci. Technol. 22:199-210.

Inokuchi, Y., Fushitani, M., Kubo, T., Sato, K. (2002) Effect of volume fraction of bundle sheath and water extractives on bending creep behavior of bamboo under changing moisture conditions. Mokuzai Gakkaishi 48:413-424 (in Japanese).

Leicester, R.H. (1971) A rheological model for mechano-sorptive deflections of beams. Wood Sci. Technol. 5:211-220.

Mukudai, J., Yata, S. (1986) Modeling and simulation of viscoelastic behavior (tensile strain) of wood under moisture change. Wood Sci. Technol. 20:335-348.

Mukudai, J., Yata, S. (1987) Further modeling and simulation of viscoelastic behavior (bending deflection) of wood under moisture change. Wood Sci. Technol. 21:49-63.

Nakano, T. (1996) A theoretical description of creep behavior during water desorption. Holzforschung 50:49-54.

Nutting, P.G. (1921) A new general law of deformation. J. Franklin Inst. 191:679-685.

Parameswaran, N., Liese, W. (1976) On the fine structure of bamboo fibres. Wood Sci. Technol. 10:231-246.

Parameswaran, N., Liese, W. (1980) Ultrastructural aspects of bamboo cells. Cellulose Chem. Technol. 14:587-609.

Schniewind, A.P. (1966) On the influence of moisture content changes on the creep of beech wood perpendicular to the grain including the effects of temperature and temperature changes. Holz Roh Werkst. 24:87-98.

Takemura, T. (1967) Plastic properties of wood in relation to the non-equilibrium states of moisture content (continued). Mokuzai Gakkaishi 13:77-81.

Takemura, T. (1968) Plastic properties of wood in relation to the non-equilibrium states of moisture content (re-continued). Mokuzai Gakkaishi 14:406-410.

Tokumoto, M., Nagae, H., Takeda, T., Nakano, T. (1998) Bending creep during moisture adsorption of wood subjected to set in bending. J. Soc. Mater. Sci. Jpn. 47:374-379 (in Japanese).

van der Put, T.A.C.M. (1989) Theoretical explanation of the mechano-sorptive effect in wood. Wood Fiber Sci. 21:219-230.

Received November 10, 2009. Accepted January 29, 2010.

Previously published online May 4, 2010. 